\title{
ARTíCULO \\ Modelación de la tasa de crecimiento de organismos filtradores en cultivo bajo limitación de alimento
}

\author{
Specific growth rate model of food limited growth of filter feeding in culture
}

Andrés Orellana-Torres ${ }^{1}$

${ }^{1}$ Los Aromos 757, Quillota, Chile. aeotorres@gmail.com

\begin{abstract}
A growth rate model is developed based on the assumption that food offer is the growth limiting factor of filter feeding in culture. For which the ingested food, defined at steady sate conditions through an energy balance, expressed as Scope for Growth terms is correlated to Specific Growth Rate through Monod model. Model give the specific growth rates as a function of environmental (seston concentration, water flow rates, fouling load), physiological (assimilation efficiency, metabolic expenditure, maintenance ration) and technological (stock density and retention efficiency of the culture system) variables. The growth rate model obtained is used to describe the growth of Venerupis pullastra seeds from data obtained under controlled conditions and simulate their behavior to changing scenarios. A full, logic and consistent description of the growth process obtained in agreement with mathematical, physiological and productive principles contribute a theoretic sustenance to aquacultural business and feasible to be applied to other species.
\end{abstract}

Key words: Energy balance, food limited growth, specific growth rate, scope for growth, suspended culture

Resumen.- Se desarrolla un modelo simple para la tasa de crecimiento de organismos filtradores en cultivo bajo el supuesto de que la oferta de alimento es el factor que limita el crecimiento. Para ello la ración ingerida, definida en condiciones de equilibrio a través de un balance energético y expresada como Potencial para el Crecimiento, se correlaciona con la Tasa de Crecimiento a través del modelo de Monod logrando asociar variables ambientales (oferta de alimento, carga de fouling, recambio de agua), fisiológicas (eficiencia de asimilación, costo metabólico, ración de mantención) y tecnológicas (eficiencia del sistema de cultivo, densidad) entre otras a las tasas de crecimiento. El modelo obtenido es utilizado para describir el crecimiento de semillas de Venerupis pullastra a partir de datos obtenidos bajo condiciones controladas y simular su comportamiento ante escenarios variables. Finalmente, se obtiene una descripción completa, lógica y consistente del proceso de crecimiento, concordante con principios fisiológicos, matemáticos y productivos que aportan sustento teórico a la actividad acuícola factible de aplicarse a cualquier especie.

Palabras clave: Balance energético, crecimiento limitado por alimento, tasa de crecimiento específico, campo para el crecimiento, cultivo suspendido

\section{INTRODUCCIÓN}

El creciente valor económico y el consecuente impacto ambiental asociado a la actividad acuícola y pesquera dedicada a la explotación de mariscos en los cuerpos de agua y de fondo marino han originado una abundante literatura sobre su fisiología (Duarte et al. 2010). Particularmente, los estudios sobre capacidad de carga, la modelación bio-económica y eco-fisiológica requieren conocer la respuesta de crecimiento de los organismos expuestos a condiciones ambientales y tecnológicas variables.

El crecimiento de los organismos bajo condiciones de cultivo es la resultante de la interacción de factores ambientales, biológicos y tecnológicos. Sin embargo, los enfoques tradicionales han conducido al desarrollo de modelos cuya aplicación en acuicultura es restringida, por cuanto no toman en cuenta el factor tecnológico, como agente modificador de la respuesta de crecimiento de los organismos cultivados (Orellana 1999).

Paloheimo \& Dickie (1965, 1966) y Ursin (1967), basados en el trabajo de Pütter (1920), desarrollaron modelos que describen el crecimiento de los peces como la diferencia entre procesos anabólicos y catabólicos logrando introducir variables tales como la oferta de alimento, tamaño del organismo, consumo de oxígeno y temperatura. Widdows (1978) utiliza la regresión múltiple para describir los efectos combinados de la concentración de alimento, el tamaño del cuerpo y la temperatura sobre el crecimiento de Mytilus edulis. Springborn et al. (1994) 
modifican el parámetro k (tasa de crecimiento) de la solución dada por Von Bertalanffy (1938) al modelo planteado por Pütter (1920), introduciendo variación estacional en el crecimiento. Más recientemente, Marambio et al. (2012) utilizaron este modelo para determinar el crecimiento de la biomasa de Mytilus chilensis en cultivo pero introduce un algoritmo para corregirlo por disponibilidad de alimento y ciclo reproductivo.

La oferta energética que las unidades filtradoras logran captar bajo condiciones de cultivo en la naturaleza (o en ambiente controlado), está sujeta a factores tales como tamaño (Camacho et al. 2000), concentración y valor energético del seston (Chauvaud et al. 1998, Arrieche 2010), velocidad y carga hidrodinámica del flujo de agua (Babarro \& Carrington 2013), carga de fouling y/o competencia espacial (Taylor et al. 1997a, Nunes et al. 2003, Marambio et al. 2012), densidad del cultivo (Taylor et al. 1997b, Fréchette \& Bacher 1998, López et al. 2010, Cubillo et al. 2012a, b) y configuración del sistema de cultivo (Márquez et al. 2011). Todos estos factores interactúan para determinar la eficiencia con que los organismos en cultivo retienen las partículas alimenticias en el sistema de cultivo.

La posterior transformación de esta materia orgánica capturada por el sistema de cultivo en energía para el crecimiento y reproducción obedece a factores fisiológicos tales como la eficiencia de asimilación (Selong et al. 2001, Babarro et al. 2003, Irisarri et al. 2013), la concentración de oxígeno (Buxton et al. 1981) y temperatura (Selong et al. 2001, Handeland et al. 2008). Esta etapa del proceso ha sido descrita inicialmente por modelos basados en el potencial para el crecimiento (SFG) donde las tasas de crecimiento se aproximan en función de la diferencia entre tasas de absorción de energía y las tasas de utilización de energía para la mantención del metabolismo (e.g., Beiras et al. 1993, 1994, Scholten \& Smaal 1998, Pérez-Camacho et al. 2000). Esta aproximación continuó evolucionado hacia los balances dinámicos de energía (DEB), modelos eco-fisiológicos más complejos incorporando procesos de partición de energía para formar reservas, tejidos y gametos (e.g., Kooijman 2000, Rueda et al. 2005, Van der Meer 2006, Van der Veer \& AllunoBruscia 2006, Rosland et al. 2009, Troost et al. 2012). Filgueira et al. (2011) compararon el desempeño de ambos modelos para explicar el crecimiento de Mytilus edulis en diferentes ambientes concluyendo que la convergencia de ambos enfoques está dominada por la conducta alimentaria y las condiciones tróficas locales, en otras palabras el crecimiento es restringido por la oferta alimentaria. De acuerdo a Duarte et al. (2012), en los modelos SFG no consideran el estado del organismo, mientras que en los modelos DEB las tasas son descritas en función del organismo y su ambiente contemplando reservas energéticas en sus diferentes tejidos. Mas recientemente, Marambio et al. (2012) buscando modelar la biomasa en cultivo de Mytilus chilensis agregan que las múltiples variables que requieren ser incorporadas y controladas impiden su aplicación a la industria.

Hirtle et al. (1981), Urban et al. (1983) y Talbot (1994) entre otros, observan como las tasas de crecimiento se relacionan con la ración alimenticia tanto en peces como en organismos filtradores. Esta relación geométrica había sido descrita por Brett et al. (1969) y su trayectoria da sustento a la hipótesis de que el crecimiento está limitado por la oferta de alimento como lo sugiere Camacho et al. (2000). Este supuesto permite encadenar factores por medio de un balance energético y por lo tanto puede ser descrito por el modelo de Monod (1942).

Conocer los mecanismos que regulan el crecimiento de los organismos y como éste es afectado por factores ambientales y de cultivo ofrece la oportunidad de manejarlo y optimizarlo. Los objetivos del presente trabajo fueron: dar sustento teórico a la actividad acuícola mediante la modelación del crecimiento desde el punto de vista de las variables críticas para el cultivo; analizar cómo esos factores interactúan entre sí y ordenar conceptos productivos utilizados comúnmente en acuicultura.

\section{Materiales y MÉTODOS}

Bajo el supuesto de que la oferta de alimento es el principal factor que limita el crecimiento de los organismos filtradores en cultivo, se realizó un balance energético a un volumen de control para relacionar entre si las variables relevantes que interactúan en un sistema de cultivo y explicar de esta manera como el sistema captura energía del medio. Este volumen de control representa el sistema o unidad de cultivo en el cual están confinados los bivalvos filtradores y que está sometido a un flujo energético entrante o saliente de seston. El flujo de alimento corresponde a un modelo de trasporte horizontal.

La siguiente etapa consistió expresar la relación obtenida previamente en forma de Potencial para el Crecimiento (SFG) bajo el supuesto de equilibrio dinámico entre demanda y oferta de alimento mediante la incorporación del concepto de energía asimilada y energía utilizada para mantención del metabolismo. 
Posteriormente, este potencial para el crecimiento capturado por los organismos en el sistema de cultivo es correlacionado con la tasa de crecimiento a través del modelo de Monod (1942), procedimiento que es explicado mediante los datos obtenidos por Brett et al. (1969).

Finalmente, se probó este modelo con datos experimentales obtenidos bajo condiciones controladas publicado por Beiras et al. (1993) para semillas de Venerupis pullastra (Montagu, 1803). Se consideró el mejor ajuste de estos datos para el modelo por el método de mínimos cuadrados logrando representarlos con un mínimo de constantes. Una vez calibrado el modelo para estos datos se analiza la sensibilidad de las tasa de crecimiento frente a variaciones de factores relevantes para el cultivo.

\section{Resultados}

\section{BALANCE ENERGÉTICO Y RACIÓN ALIMENTICIA INGERIDA} A continuación se analiza cómo la ingesta o demanda de alimento se relaciona con la oferta de alimento, bajo condiciones de equilibrio. Para ello se considera el volumen de control de la Fig. 1, que representa una unidad de cultivo (pearl net, linterna, raft) cuya superficie $S\left(\mathrm{~m}^{2}\right)$ está expuesta al flujo de agua cuya carga o concentración de seston es $[S e s t]_{E}\left(\mathrm{~J} \mathrm{~m}^{-3}\right)$ que entra a una tasa de $V_{E}\left(\mathrm{~m}^{3}\right.$ $\mathrm{h}^{-1}$ ) y deja el sistema a una velocidad y carga de seston de $V_{S}\left(\mathrm{~m}^{3} \mathrm{~h}^{-1}\right)$ y $[\text { Sest }]_{S}\left(\mathrm{~J} \mathrm{~m}^{-3}\right)$, respectivamente.

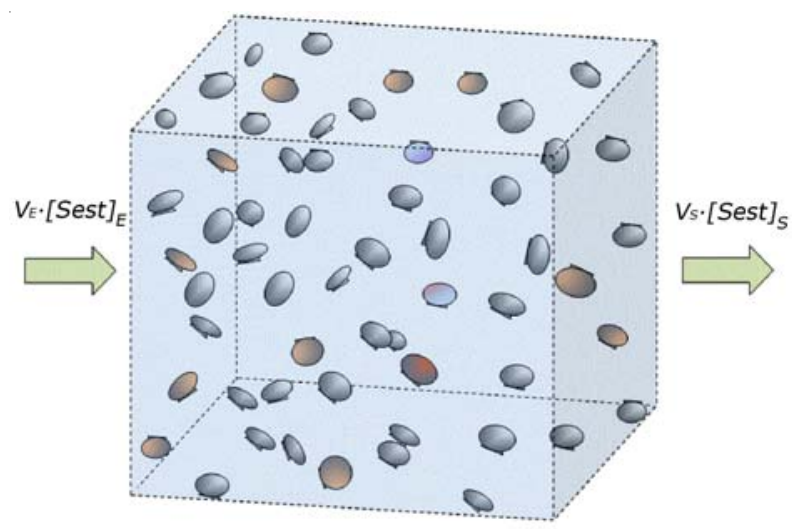

Figura 1. Volumen de control. $\mathrm{V}_{\mathrm{E}^{\prime}}$ [Sest $]_{\mathrm{E}}$ y $\mathrm{V}_{\mathrm{S}^{\prime}}[\text { Sest }]_{\mathrm{S}}$ velocidad del flujo y concentración de alimento a la entrada y salida del volumen de control, respectivamente / Control volume. $V_{E^{\prime}}$ [Sest $]_{E}$ and $V_{S^{\prime}}$ $[\text { Sest }]_{S}$ flow rate and food load at entry and outlet of control volume
Dentro de este volumen de control se encuentran homogéneamente distribuidas $D o$ unidades de organismos filtradores por $\mathrm{m}^{2}$ (ufilt $\mathrm{m}^{-2}$ ), cuya tasa de ingestión es Tio unidades de energía ( $\mathrm{J} \mathrm{h}^{-1}$ ufilt $^{-1}$ ) y $D$ f unidades de fouling por $\mathrm{m}^{2}$ (ufoul $\mathrm{m}^{-2}$ ), que consumen Tif unidades de energía $\left(\mathrm{J} \mathrm{h}^{-1}\right.$ ufoul $\left.^{-1}\right)$. Al realizar un balance energético se obtiene que:

Energía entrante $=$ Energía demandada por unidad filtradora + Energía demandada por unidad de fouling + Energía saliente

$V_{E} \cdot S \cdot[\text { Sest }]_{E}=D_{o} \cdot S \cdot T i o+D_{f} \cdot S \cdot T i_{f}+V_{s} \cdot S \cdot[\text { Sest }]_{s}$

Si se considera que el sistema está en equilibrio dinámico, entonces una fracción $\eta$ de la oferta energética entrante al sistema está siendo retenida, de manera que:

$$
\eta \cdot V_{E} \cdot S \cdot[\text { Sest }]_{E}=D_{o} \cdot S \cdot T i_{o}+D_{f} \cdot S \cdot T i_{f}
$$

Debido a que la variable espacial $S$ es una constante para los 3 términos en ec. (3) al simplificarla y ordenarla se obtiene:

$$
\eta \cdot V_{E} \cdot[\text { Sest }]_{E}-D_{f} \cdot T i_{f}=D o \cdot T i o
$$

En (4) se denomina al término a la izquierda de la igualdad como la oferta neta de alimento hacia el sistema (ONA). Luego, la oferta neta de alimento ingerida por cada unidad filtradora en el sistema corresponde a:

$$
\frac{\eta \cdot V_{E} \cdot[\text { Sest }]_{E}-D_{f} \cdot T i_{f}}{D_{o}}=\text { Tio }
$$

Finalmente, si se expresa la oferta o demanda de alimento en proporción al tamaño del organismo $(\omega)$ esto corresponde a la tasa específica de alimentación (SFR, specific food rate) utilizada por los productores:

$$
S F R=\frac{T i o}{\omega}
$$

La tasa específica de alimentación en el equilibrio equivale a la oferta neta de alimento que captura el sistema cultivo y es igual al consumo de las unidades filtradoras en el sistema para cada tamaño. La configuración del sistema de cultivo queda definida a través de su eficiencia de retención $(\eta)$, cuyo valor reciproco es el Factor de Conversión $(F C)$ utilizado por los cultivadores. Mediante un sencillo análisis se ha logrado asociar tasa de ingestión con variables tales como el tamaño, la densidad, la carga de fouling, el flujo de alimento que puede ser separado en sus componentes: tasa de ingreso, concentración y valor nutricional o energético. 


\section{RACIÓN ALIMENTICIA ASIMILADA: OFERTA Y DEMANDA DE POTENCIAL PARA EL CRECIMIENTO}

El análisis previo nos permite explicar la ingesta del material particulado bajo condiciones de equilibrio dinámico en un sistema de cultivo.

El potencial para el crecimiento de un organismo (SFG, scope for growth), es la diferencia entre la energía asimilada a través de procesos de digestión y la liberada durante los proceso de mantención de su metabolismo para cada tamaño y temperatura (Parsons et al. 1995, Handeland et al. 2008). Cuando el nivel energético del organismo supera el de su metabolismo de mantención es esperable observar incrementos en la tasa de crecimiento.

En el presente modelo, el potencial para crecer se obtiene multiplicando ambos miembros de la ec. (5) por la eficiencia de asimilación (EA) y luego se descuenta el costo metabólico. Este último es evaluado como la ración de mantención $(R m)$, cuyo nivel energético es equivalente a la suma de las tasas respiratoria y excretoria principalmente:

$$
E A \cdot \frac{\left(\eta \cdot V_{E} \cdot[\text { Sest }]_{E}-D_{f} \cdot \text { Tif }\right)}{D_{o}}-R m=E A \cdot T i o-R m
$$

El término a la izquierda de la igualdad en (7) representa la oferta de $S F G$ y el término a la derecha representa la demanda de $S F G$. En términos de la tasa específica de alimentación y de la ración de mantención expresada en función del tamaño del organismo (ración de mantención específica) se puede reescribir la ec. (7) como sigue:

$S F G=E A \frac{\left(\eta \cdot V_{E} \cdot[\text { Sest }]_{E}-D_{f} \cdot T i_{f}\right)}{D o \cdot \omega}-\frac{R m}{\omega}=E A \cdot S F R-\frac{R m}{\omega}(8)$

La aproximación encontrada indica que bajo condiciones de equilibrio, la energía que el organismo podría destinar a crecer (y/o reproducirse), equivale a la que eficientemente asimila del medio menos la que destina a la mantención de su metabolismo. El lado derecho de este equilibrio establece una relación lineal entre $S F G$ y $S F R$ (ec. 8), con pendiente $E A$ e intersección $-R m / \omega$.

\section{LA CURVA DE BRETT Y EL MODELo de Monod}

Brett et al. (1969) evaluaron las tasas de crecimiento de Oncorhynchus nerka frente a diferentes raciones alimenticias. En la curva descrita por estos autores para cada temperatura y tamaño (Fig. 2), la trayectoria que realiza la tasa específica de crecimiento ( $S G R$, specific growth rate) de un organismo frente a sucesivos incrementos en la ración alimenticia (SFR) hasta un valor

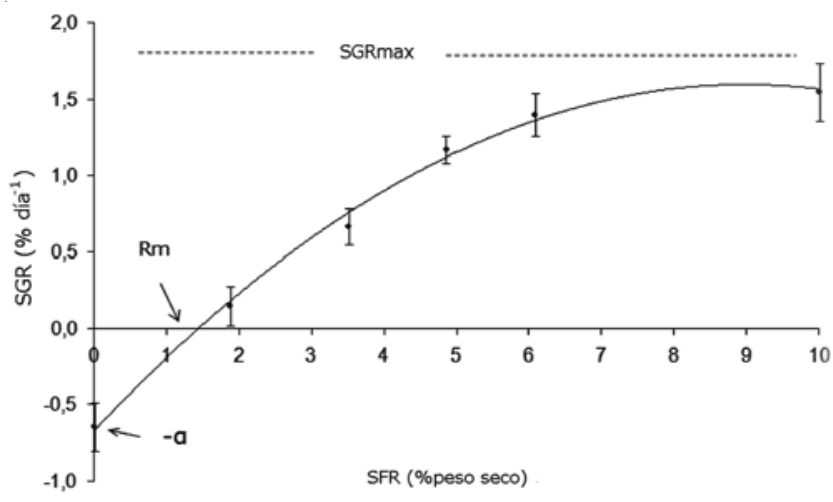

Figure 2. Tasa específica de crecimiento (\% día $\left.{ }^{-1}\right)$ obtenida por Brett et al. (1969) para Oncorhynchus nerka de 6,0 g bajo diferentes raciones alimenticias (\% peso seco) a $15^{\circ} \mathrm{C} /$ Specific growth rate (\% day ${ }^{-1}$ ) of young sockeye salmon (Oncorhynchus nerka) $6 \mathrm{~g}$ in relation to diferent ration (\% dry weigth) at $15^{\circ} \mathrm{C}$, obtained by Brett et al. (1969)

asintótico queda definida por 3 puntos: (1) El intercepto con la ordenada ( $-a)$, que representa la velocidad a la que el organismo pierde peso bajo raciones nulas de alimento; el estado energético asociado es conocido como metabolismo de inanición. (2) El intercepto con el eje de abscisas $(R m)$, que representa la ración de alimento en la que el incremento de peso es nulo; el estado energético asociado es denominado metabolismo de mantención. (3) La tasa de crecimiento asintótica (SGRmax), donde la tasa de crecimiento se vuelve independiente de la oferta de alimento.

Desde el punto de vista energético se puede expresar los datos obtenidos por estos autores para la oferta de alimento en función del potencial para crecer (SFG) a partir de la relación lineal existente entre $S F G$ y $S F R$ mencionada anteriormente. De esta manera si se traza en un gráfico de Lineweaver-Burk, cuya trasformación permite linealizar la relación entre 2 variables, los valores recíprocos de la tasa específica de crecimiento y de las raciones ofertadas obtenidas por Brett et al. (1969) expresadas como SFG, se obtiene una recta cuya ecuación es (Fig. 3):

$$
\frac{1}{S G R}=\frac{1}{S G R \max }+m \cdot \frac{1}{S F G}
$$

donde 1/SGRmax representa la intersección con el eje de las ordenadas y $m$ es la pendiente. Sumando ambos términos a la derecha de (9) se tiene: 


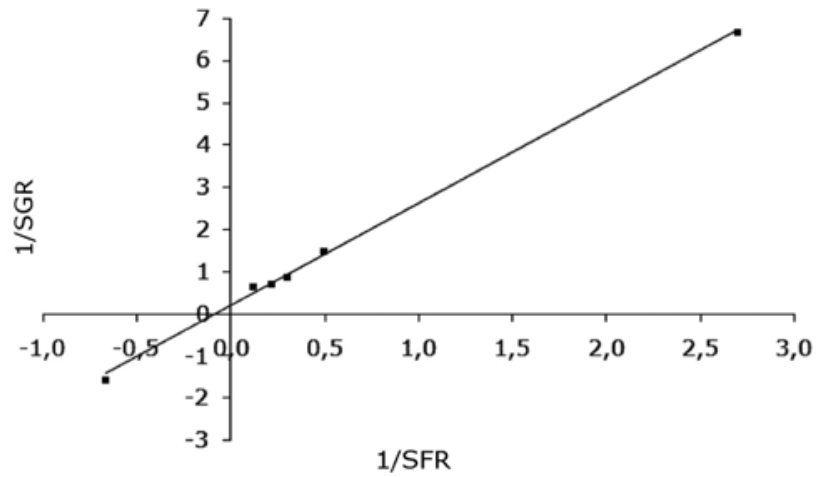

Figura 3. Gráfico de Lineweaver-Burk o de recíprocas doble para los datos observados por Brett et al. (1969) (Fig. 3). Véase Robertis \& Robertis (1984) para mayores detalles / Lineweaver-Burk plot to Brett et al. (1969) obtained data (Fig. 3). See Robertis \& Robertis (1984)

$$
\frac{1}{S G R}=\frac{S F G+m \cdot S G R \max }{S G R \max \cdot S F G}
$$

En (10) $K_{S}=m \cdot S G R m a x$. Invirtiendo esta última ecuación e introduciendo el resultado (8) para $S F G$, se obtiene el modelo de Monod (1942) cuyo origen está desplazado $R m / \omega$ unidades hacia la izquierda (Fig. 4).

$$
S G R=S G R \max \cdot \frac{E A \cdot S F R-R m / \omega}{K S+E A \cdot S F R-R m / \omega}
$$

La relación anterior explica el crecimiento de los organismos cuando el alimento es el factor limitante. Ordenando y expandiendo $S F R$ en esta última expresión, se obtiene el modelo de crecimiento buscado

$S G R=S G R \max \cdot \frac{E A \cdot\left(\eta \cdot V_{E} \cdot[\text { Sest }]_{E}-D_{f} \cdot T i_{f}\right)-D o \cdot R m}{D_{o} \cdot K s+E A \cdot\left(\eta \cdot V E \cdot[\text { Sest }]_{E}-D_{f} \cdot T i_{f}\right)-D o \cdot R m}$

\section{Determinación de Ks}

Cuando la ración ingerida es nula $(S F R=0)$ el organismo recurre a sus reservas energéticas para mantener su metabolismo basal perdiendo peso a una velocidad de - $a$ \% día ${ }^{-1}$, en una razón proporcional a su tamaño y a la temperatura entre otros factores. De este modo, reemplazando en (11) $S F R=0$ y $S G R=-a$ :

$$
-a=S G R m a x \cdot \frac{-R m / \omega}{K s-R m / \omega}
$$

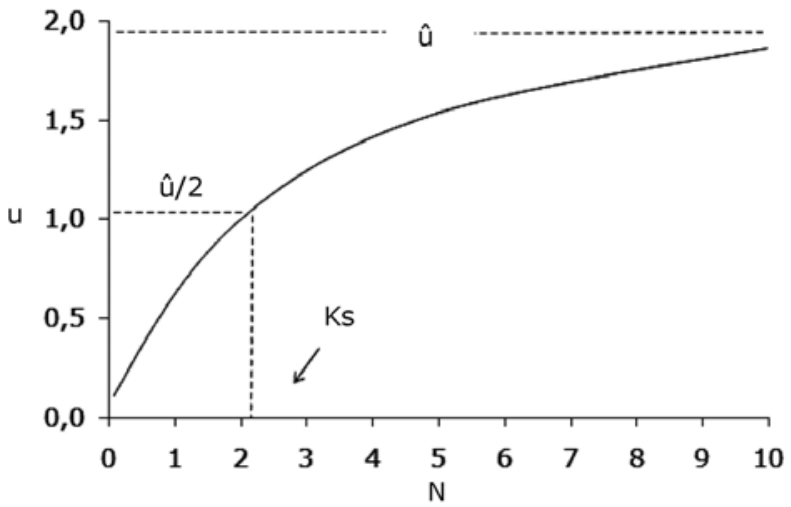

Figura 4. Descripción gráfica del modelo de Monod. $\mathrm{u}=$ tasa de crecimiento $\left(\%\right.$ día $\left.^{-1}\right) ; \hat{u}=$ tasa de crecimiento asintótica $\left(\%\right.$ día $\left.^{-1}\right) ; \mathrm{N}$ = concentración del nutriente limitante $\left(\mathrm{mg} \mathrm{L}^{-1}\right)$ y $\mathrm{K}_{\mathrm{s}}=$ contante de saturación media. Ver Parsons et al. (1995) / Monod model graphic description. $\mathrm{u}=$ growth rate $\left(\%\right.$ day $\left.^{-1}\right)$; $\mathrm{u}=$ asymptotic growth rate $\left(\%\right.$ day $\left.{ }^{-1}\right) ; \mathrm{N}=$ limitant nutrient concentration $\left(\mathrm{mg} \mathrm{L}^{-1}\right)$ and $\mathrm{K}_{\mathrm{s}}=$ half saturation coefficient. See Parsons et al. (1995)

Despejando $K_{S}$ de (13) se obtiene:

$$
K s=\left(\frac{S G R \max }{a}+1\right) \cdot R m / \omega
$$

$K_{S}$, en el modelo de Monod o la constante de MichaelisMenten es un parámetro característico que corresponde a la concentración del nutriente limitante en que se obtiene la mitad de la tasa de crecimiento asintótica, su valor es un indicador de la afinidad de un microorganismo por determinado nutriente. Análogamente en este modelo, $K_{S}$ es un múltiplo de $R m \geq 1$, que representa la oferta energética en que se obtiene la mitad de la tasa de crecimiento asintótica ( $1 / 2$ SGRmax) de un organismo.

\section{Aplicación DEL MODElo}

Beiras et al. (1993) mantuvieron semillas de Venerupis pullastra de 2,0 mm (0,078 mg de peso orgánico), a una densidad (Do) de 200 semillas por recipiente de $250 \mathrm{ml}$, en los cuales se mantenía un flujo continuo de agua $\left(V_{E}\right)$ de $0,13 \mathrm{~L} \mathrm{~h}^{-1}$ cuya concentración de Isochrysis galbana

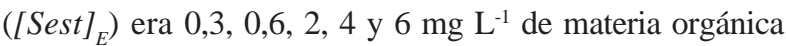
equivalentes a $15,30,100,200,300 \mathrm{cel} \mathrm{l}^{-1}$, respectivamente. El flujo se regulaba de manera que la concentración de células nunca excedía el 25\% entre el ingreso y la salida del set experimental ( $\eta \geq 75 \%$ ). Si se considera que la carga de fouling es nula $\left(D_{f}=0\right)$. La Tabla 1 presenta las tasas de crecimiento en peso vivo (SGR) y eficiencias de asimilación (EA), observadas frente a diferentes ofertas de alimento $\left(V_{E} \cdot[\text { Sest }]_{E}\right)$ a una temperatura de $19 \pm 1^{\circ} \mathrm{C}$. 
Tabla 1. Tasas de crecimiento y eficiencias de asimilación observadas por Beiras et al. (1993) frente a diferentes ofertas de alimento / Growth rates and assimilation efficiency observed respect different food given by Beiras et al. (1993)

\begin{tabular}{ccc}
\hline $\begin{array}{c}V_{E} \cdot[\text { Sest }]_{E} \\
\left(\mathrm{mg} \mathrm{h}^{-1}\right)\end{array}$ & $E A$ & $\begin{array}{c}S G R \\
\left(\% \mathrm{dia}^{-1}\right)\end{array}$ \\
\hline 0,039 & 0,87 & 0,5 \\
0,078 & 0,84 & 1,7 \\
0,260 & 0,81 & 7,0 \\
0,520 & 0,73 & 9,2 \\
0,780 & 0,61 & 11,6 \\
\hline
\end{tabular}

En este caso no hay fouling, además la eficiencia de asimilación decrece en forma lineal con respecto a la concentración de alimento de acuerdo a:

$$
E A=0,9-0,3 \cdot\left(V_{E} \cdot[\text { Sest }]_{E}\right)
$$

Por lo tanto al combinar este resultado con el modelo simplificado obtenido en ec. 12 se obtiene:

$$
S G R=S G R \max \cdot \frac{\eta \cdot\left[0,9 \cdot V_{E} \cdot[\text { Sest }]_{E}-0,3 \cdot\left(V_{E} \cdot[\text { Sest }]_{E}\right)^{2}\right]-D o \cdot R m}{D o \cdot K s+\eta \cdot\left[0,9 \cdot V_{E} \cdot[\text { Sest }]_{E}-0,3 \cdot\left(V_{E} \cdot[\text { Sest }]_{E}\right)^{2}\right]-D o \cdot R m}
$$

Cuyas incógnitas son: la tasa de crecimiento asintótica (SGRmax), la constante de saturación $(\mathrm{Km})$ y la ración de mantención $(R m)$. Al iterar la ec. 16 con los valores de la Tabla 1, se encuentran los siguientes resultados para los parámetros:

SGRmax $=25 \pm 2 \%$ día $^{-1}$.

$K s=0,0023 \pm 0,0005 \mathrm{mg} \mathrm{h}^{-1}$ que corresponden a 200 cel $\mu \mathrm{l}^{-1}$.

$R m=0,000044 \pm 0,000005 \mathrm{mg} \mathrm{h}^{-1}$ que corresponden a 5 cel $_{\mu \mathrm{l}^{-1}}$.

Además, las intersecciones con el eje de las ordenadas son $\mathrm{X}_{1}=0,013$ y $\mathrm{X}_{2}=2,8 \mathrm{mg} \mathrm{h}^{-1}\left(5 \mathrm{y} 1146 \mathrm{cel} \mu \mathrm{l}^{-1}\right.$ ), respectivamente y la máxima tasa de crecimiento de $13,07 \%$ día ${ }^{-1}$ se obtiene a $1,48 \mathrm{mg} \mathrm{h}^{-1}\left(569\right.$ cel $\left.\mu \mathrm{l}^{-1}\right)$, aproximadamente (Fig. 5). A partir de esta información se puede deducir que las semillas bajo inanición perderán peso una tasa de: $a=-0,49 \%$ día $^{-1}$.

\section{EFECTO DE FACTORES SOBRE TASAS DE CRECIMIENTO DE $\boldsymbol{V}$. PULLASTRA}

La figuras 6, 7, 8 y 9 muestran el efecto sobre las tasa de crecimiento de $V$. pullastra para variaciones en la cantidad de fouling ( $D f$ Tif), la eficiencia o configuración del sistema de cultivo $(\eta)$, la densidad $(D o)$ y la dieta $(E A)$. Estas se elaboraron a partir del modelo completo (ec. 12) con los

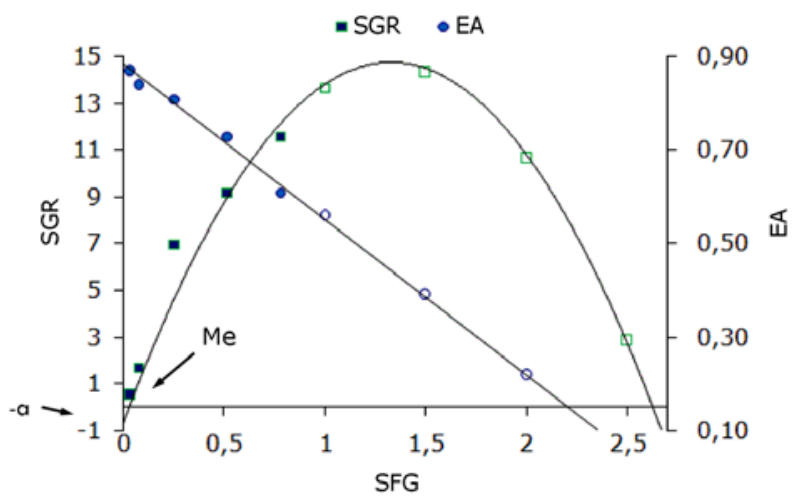

Figura 5. Tasa de crecimiento (SGR) para diferentes potenciales para el crecimiento (SFG) observadas por Beiras et al. (1993). $\mathrm{Rm}=$ costo metabólico $\left(\mathrm{mg} \mathrm{h}^{-1}\right)$; $-a=$ tasa pérdida de peso $\left(\%\right.$ día $\left.^{-1}\right)$, EA= Eficiencia de asimilación / Growth rate (SGR) and scope for growth (SFG) obtained by Beiras et al. (1993). Rm= metabolic expenditure $\left(\mathrm{mg} \mathrm{h}^{-1}\right) ;-a=$ loss weight rate $\left(\%\right.$ day $\left.^{-1}\right) ; \mathrm{EA}=$ Assimilation efficiency

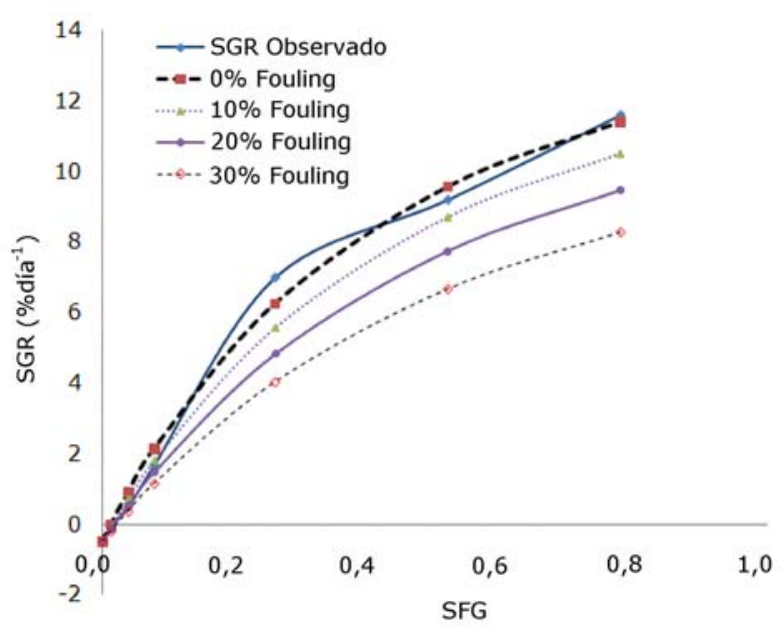

Figura 6. Efecto de variaciones en la cantidad de fouling asociada a la unidad de cultivo sobre las tasas de crecimiento de Venerupis pullastra / Simulation of Venerupis pullastra growth rates under varying amount of fouling 
parámetros o constantes obtenidas para V. pullastra bajo las condiciones mantenidas por Beiras et al. (1993).

Para estimar el efecto del fouling asociado a la unidad de cultivo sobre las tasas de crecimiento de V. pullastra (Fig. 6), se simuló un consumo de alimento equivalente al 0, 10, 20, 30 de lo disponible para las semillas. La simulación arrojó una significativa disminución de la tasa de crecimiento de las semillas de $V$. pullastra frente al aumento en la cantidad de fouling y su competencia por el alimento.

Para estimar el efecto de variaciones en la eficiencia sistema cultivo o su configuración sobre las tasas de crecimiento de V. pullastra (Fig. 7) se simuló en el modelo eficiencias del 90; 75; 60 y 50\%, respectivamente bajo nula presencia de fouling. La simulación arrojó una significativa disminución de la tasa de crecimiento de las semillas de $V$. pullastra frente a disminuciones en la eficiencia del sistema de cultivo.

Para estimar el efecto de variaciones en la densidad de cultivo sobre las tasas de crecimiento de V. pullastra (Fig. 8), se modificó el modelo para densidades de 300, 200, 100 y 50 unidades filtradoras, respectivamente. La simulación arrojó un aumento significativo e importante de la tasa de crecimiento frente a disminuciones en las densidades de cultivo.

Para estimar el efecto de variaciones en la eficiencia de asimilación o dieta sobre las tasas de crecimiento de $V$. pullastra (Fig. 9) se simuló eficiencias de asimilación

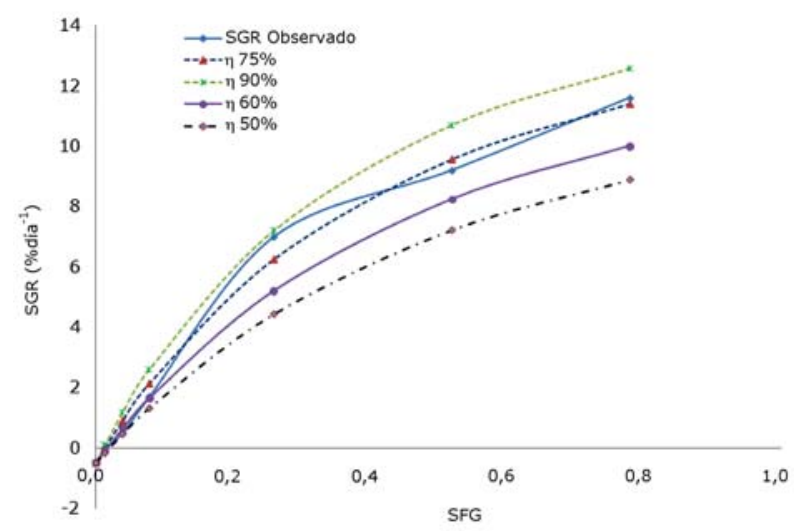

Figura 7. Efecto de variaciones en la eficiencia sistema cultivo (configuración) $\boldsymbol{h}$ sobre las tasas de crecimiento de Venerupis pullastra / Simulation of Venerupis pullastra growth rates under varying efficiency of culture system $(h)$

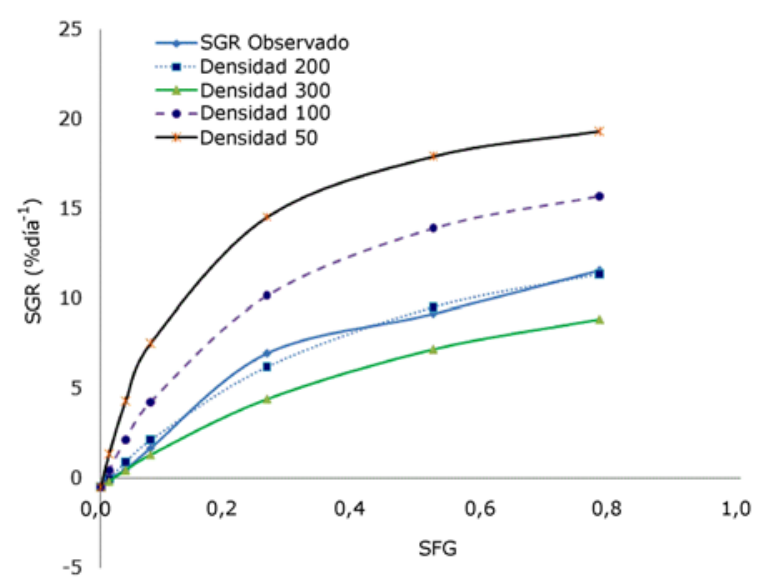

Figura 8. Efecto de variaciones en la densidad de cultivo sobre las tasas de crecimiento de Venerupis pullastra / Simulation of growth rates Venerupis pullastra under variable culture density

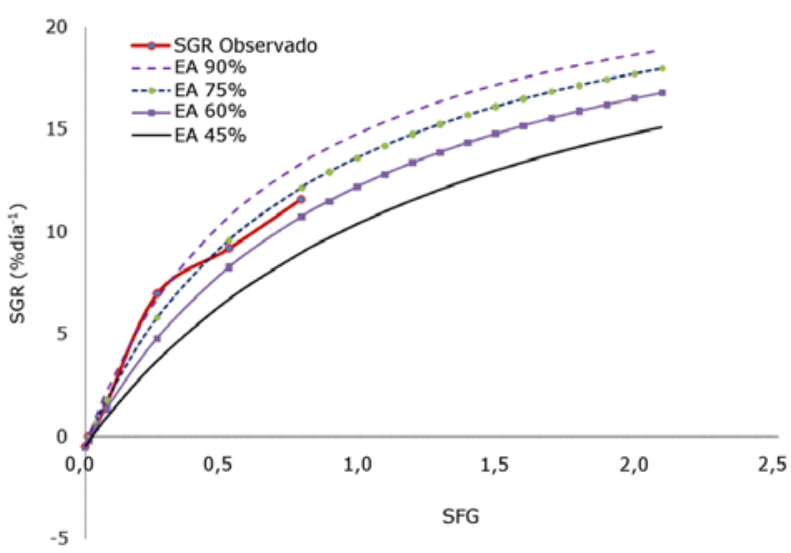

Figura 9. Efecto de variaciones en la eficiencia de asimilación (dieta) sobre las tasas de crecimiento de Venerupis pullastra / Simulation of growth rates Venerupis pullastra under a variable assimilation efficiency

equivalentes a 90, 75, 60 y 45\%, respectivamente. La simulación arrojó un aumento significativo de la tasa de crecimiento de las semillas de $V$. pullastra frente a aumentos en las eficiencias de asimilación.

\section{Discusión}

Se ha desarrollado una expresión para la tasa de crecimiento bajo el supuesto de que la oferta de alimento es el factor limitante del crecimiento de organismos filtradores bajo cultivo suspendido. La condición 
necesaria y suficiente para calibrar el modelo es el equilibrio entre la oferta y la demanda energética, condición que en cultivo generalmente ocurre durante períodos invernales o bajo exceso de densidad y carga de fouling. Estos resultados coinciden con los de Camacho et al. (2000), quienes concluyen que las diferencias observadas en crecimiento para idénticos tamaños son explicados por limitación en el alimento y el espacio disponible, como consecuencia de la aglomeración de Mytilus galloprovincialis en cultivo. Cubillo et al. (2012 a, b) y Marambio et al. (2012) agregan que también la competencia intra-específica e interespecífica por el espacio y el alimento explican sus resultados.

Se logra incorporar, a través de un análisis sencillo, variables tales como carga de seston, carga de fouling, densidad de cultivo, velocidad del flujo de agua (tasa de recambio), eficiencia de retención del sistema de cultivo (configuración), que son factores de gran importancia en el proceso de diseño, planificación de un sistema de cultivo y en la estimación de la capacidad de carga de los sistemas acuáticos.

En este trabajo se ha optado por utilizar el modelo de Monod para expresar la tasa de crecimiento, una vez asociadas las variables relevantes y expresado el potencial para el crecimiento bajo equilibrio dinámico. La sencillez y congruencia con las variables fisiológicas del modelo de Monod resulta conveniente para la búsqueda de soluciones matemáticas y determinar el efecto que tiene un determinado set de variables sobre el crecimiento.

En este sentido y a diferencia de las tradicionales correlaciones y modelos de crecimiento o eco-fisiológicos este estudio explica la etapa previa o de captura de alimento, mientras los modelos SFG o DEB son poderosos en explicar las etapas posteriores asociadas a la asimilación, la formación de las reservas energéticas y tejidos del organismo, sin aportar precisión sobre los mecanismos que regulan la oxidación de la materia orgánica para liberar su energía, donde la presencia y/o captura de la oferta de oxígeno para organismos aeróbicos o que realizan el ciclo de Krebs establece otra fuente de limitación para el crecimiento y donde procesos moleculares como la formación de ATP y el proceso de transcripción del ARN dependen además de un pool de nutrientes que pueden tornase limitantes bajo ciertas circunstancias.

En todos los casos mencionados existen procesos dominados por factores limitantes y esta la principal razón por la que los modelos independiente de su complejidad u origen convergen dentro de ciertos rangos tal como lo demuestra Filgueiras et al. (2011) a través de ajustar la constante de Michaelis-Menten para la tasas de ingestión de los modelos evaluados. La convergencia también se manifiesta en los valores potenciales o asintóticos de variables tales como la tasa de ingestión o asimilación, los cuales son castigados o corregidos de acuerdo a los valores que toman las variables de ambos modelos.

De acuerdo a Marambio et al. (2012) los modelos SFG y DEB no resultan prácticos en ambiente natural debido a las múltiples variables que requieren ser incorporadas y controladas, lo que impide que sean aplicados a la industria de cultivo. Particularmente, en DEB parece difícil explicar que sucederá bajo una depleción de oxígeno debido a la excesiva parametrización y se pierde la perspectiva ponderando en exceso la variable temperatura.

En síntesis, los mecanismos involucrados en el proceso de crecimiento y que sustentan el modelo presentado son: captura del alimento; asimilación y oxidación de la materia orgánica, todos los cuales son susceptibles de ser descritos mediante la cinética enzimática y el modelo de Monod. Aunque no se ha descrito explícitamente la limitación del crecimiento por la oferta de oxígeno, se ha sugerido un tratamiento similar al que se realiza con la oferta de alimento adicional al que está implícito en la determinación del costo de mantención en SFG y en DEB. Una manera más formal y concreta de representar esta idea es a través de la siguiente expresión:

$$
\frac{\delta \omega}{\delta t}=\operatorname{Vmax} \cdot \frac{f_{1}}{K f_{1}+f_{1}} \cdot \frac{f_{2}}{K f_{2}+f_{2}} \cdot \cdots \cdot \frac{f_{n}}{K f_{n}+f_{n}}=\operatorname{Vmax} \prod_{i=1}^{n} \frac{f_{i}}{K f_{i}+f_{i}}
$$

donde, la tasa de crecimiento $(\delta \omega / \delta t)$, está sujeta a la tasa máxima Vmax, corregida por la productoria de componentes limitantes fi para el cual existe su correspondiente constante de Michaelis-Menten Kfi que lo caracteriza.

De acuerdo a la simulación el crecimiento de las semillas de $V$. pullastra, es castigado al aumentar la densidad de cultivo y la competencia por alimento (fouling); mientras que mejoras en la eficiencia del sistema de cultivo y en la dieta mejoraron las tasas de crecimiento de estas semillas. En el modelo la trayectoria que describe la tasa de crecimiento tiende a una asíntota. Sin embargo, la trayectoria de la tasa de crecimiento extrapolada del ejemplo de Beiras et al. (1993) es parabólica debido a que la eficiencia de asimilación ( $E A)$ es una función decreciente de la concentración de seston. De lo anterior se observa que primero existe una $S G R$ máxima o 
asintótica y segundo existe un nivel o ración energética asimilada que maximiza la $S G R$ (ración óptima).

Riisgard \& Randlov (1981), Navarro \& Winter (1982), Urban et al. (1983) y Beiras et al. (1993, 1994), entre otros, obtienen resultados de crecimiento en bivalvos filtradores similares a los obtenidos por Brett et al. (1969) y Xie et al. (1997) en peces y por Hirtle et al. (1981) en crustáceos. Esto confirma el supuesto básico de que el crecimiento en estas especies es limitado por la oferta alimenticia como lo proponen Rosemberg \& Loo (1983), Rheault \& Rice (1993), Pilditch et al. (1996), Raillard \& Ménesguen (1994), Scholten \& Smaal (1998) y Yi (1998), entre otros autores. No obstante, Buitrago et al. (2009) no encontraron correlación en el crecimiento de Crassostrea rhizophora pero manifiesta que el alimento no fue limitante.

Como se anticipó, los factores ambientales, fisiológicos y tecnológicos interactúan para determinar un valor de eficiencia de retención $(\eta)$. Un sistema de cultivo en particular puede ser caracterizado conociendo su configuración espacial que es la que determina la capacidad de capturar y distribuir la oferta energética hacia las unidades filtradoras. Su estimación necesariamente debe hacerse indirectamente a través de ajustar datos observados de crecimiento frente a diferentes ofertas de alimento.

El balance energético plantea una distribución homogénea de las unidades filtradoras dentro del volumen de control. Salvo excepciones, el cultivo tradicional en sistema suspendido se podría describir como una sobreposición de pisos o aglomeración donde las unidades filtradoras están generalmente hacinadas. Consecuentemente, mientras más homogéneamente distribuidas estén en la columna de agua las unidades filtradoras, mayor será la probabilidad de que logren captar las partículas alimenticias incrementándose el campo para el crecimiento y por lo tanto la eficiencia de retención del sistema de cultivo. Esta es la clave en el diseño de las estructuras de cultivo.

En el balance de energía se incluyó la variable $V_{E}$ (velocidad del flujo entrante) $\left(\mathrm{m} \mathrm{h}^{-1}\right)$, esta variable confiere la calidad de flujo a la carga de seston por lo tanto puede ser reemplazada por unidades de volumen tiempo $\left(\mathrm{m}^{3} \mathrm{~h}^{-1}\right)$.

En cultivo de moluscos el crecimiento generalmente es evaluado como incrementos en longitud por unidad de tiempo $(\delta / / \delta t)$, de acuerdo a Ricker (1958). Sin embargo, Riisgard \& Randlov (1981), Urban et al. (1983) y Brown \& Hartwick (1988) observan que a raciones bajas o nulas de alimento las tasas de crecimiento en longitud de diferentes bivalvos filtradores experimentan cierto incremento a costa del consumo de reservas energéticas almacenadas en diferentes tejidos (manto, gónada, músculo). Este factor de incerteza hace no recomendable el uso de tasas de crecimiento en longitud para efectos de calibrar los modelos.

Un fenómeno común observado durante períodos de bloom de fitoplancton en cultivo suspendido de moluscos es el rápido hundimiento de las líneas de cultivo. Tal fenómeno puede ser explicado por el modelo como el resultado de un incremento rápido en el peso que experimentan los organismos bajo cultivo y por lo tanto de las estructuras que los sostienen con la consecuente ruptura del equilibrio hidrostático del sistema. La presencia de una excesiva carga de fouling hace que este fenómeno sea más notorio.

Algunas otras fuentes de error o excepciones al modelo desarrollado son (1) la estrategia fisiológica que se observa en organismos sometidos a una baja o nula oferta energética es la reducción de su metabolismo y por tanto del costo energético asociado, por lo tanto la descripción matemática de la misma en el modelo podría dar origen a un valor diferente de $-a$. (2) El proceso de formación y liberación de gametos da origen a variabilidad sobre las tasas de crecimiento en la medida que el organismo crece y destina una mayor proporción de sus reservas energéticas a gametogénesis, por lo tanto la correlación en el modelo tenderá a decaer. Este proceso de estancamiento del crecimiento también se observa cuando los organismos en cultivo sufren procesos infecciosos. (3) El fouling, además de competir por la oferta energética, reduce la carga hidrodinámica del flujo entrante (Taylor et al. 1997). Este efecto también lo causa el enmallado de diámetro variable de las estructuras de cultivo, alterando por lo tanto la velocidad del flujo entrante al sistema (Cole et al. 1996, Penney \& Mills 1996). (4) Cualquier alteración del entorno implica un estrés que el organismo debe compensar recurriendo tanto a mecanismos pasivos como a mecanismos activos. El costo energético de este estrés ambiental se manifiesta en el campo para el crecimiento que es sensible respecto a factores tales como la cantidad y calidad de alimento, temperatura y salinidad (Navarro \& Thompson 1996, Navarro \& Gonzalez 1998). Cada factor estresante actúa a diferentes niveles en el modelo, por lo que su inclusión requiere expresar las variables pertinentes en función de este factor, por ej. la tasa respiratoria como función de la temperatura y la salinidad. Babarro \& Carrington (2013) proponen que la estrategia fisiológica de Mytilus galloprovincialis para enfrentar la exposición a corrientes y oleaje es minimizar su exposición 
reduciendo su tamaño y aumentando la cantidad y el espesor de los visos sacrificando crecimiento. (5) Durante el desarrollo del modelo el balance energético se hace tomando en cuenta que existe un flujo de material particulado cuyo contenido energético está en directa relación a la calidad y cantidad de seston, lo cual es consistente con lo que ocurre en la naturaleza. Sin embargo, no todo el seston es material orgánico aprovechable y su valor energético es variable, en la naturaleza además se encuentra a menudo con material en suspensión de bajo valor energético (Winter 1977, Widdows et al. 1979, Bayne et al. 1989) que el organismo compensa con mecanismos selectivos, regulando la apertura de valvas, la tasa de filtración o bombeo de agua, el rechazo pre-ingestivo y/o producción de pseudoheces. Según algunos autores, los bivalvos filtradores pueden ingerir de forma selectiva y/o digerir alimentos diferentes al mismo tiempo de hacer ajustes para maximizar la utilización de partículas ricas en clorofila (Duarte et al. 2010, Irisarri 2013). (6) La oferta de oxígeno puede ser un factor limitante para el crecimiento. Un modelo más complejo y más completo puede incluir su efecto de la misma manera como fue descrito en este trabajo.

\section{Agradecimientos}

Se agradece al Dr. Mauricio González Abrigo de la Universidad Católica de la Santísima Concepción y al Dr. Sergio Zúñiga Jara de la Universidad Católica del Norte, quienes colaboraron con sus consejos al mejoramiento del presente trabajo.

\section{LITERATURA CITADA}

Arrieche D. 2010. Efecto del flujo del seston sobre la fisiología energética del callo de hacha Atrina maura (Sowerby 1835) y del mejillón de laguna Mytella strigata (Hanley 1843). Tesis Doctorado, Centro de Investigaciones Biológicas del Noreste, S.C, La Paz, 169 pp.

Babarro J \& E Carrington. 2013. Attachment strength of the mussel Mytilus galloprovincialis: Effect of habitat and body size. Journal of Experimental Marine Biology and Ecology 443: 188-196.

Babarro J, M Fernández-Reiriz \& U Labarta. 2003. In situ absorption efficiency processes for the cultured mussel Mytilus galloprovincialis in Ría de Arousa (northwest Spain). Journal of the Marine Biology Association of the United Kingdom 83: 1059-1064.

Bayne B, A Hawkins, E Navarro \& I Iglesias. 1989. Effects of seston concentration on feeding, digestion and growth in the mussel Mytilus edulis. Marine Ecology Progress Series 55: 47-54.
Beiras R, A Perez \& M Albentosa. 1993. Influence of food concentration on energy balance and growth performance of Venerupis pullastra seed reared in an open-flow system. Aquaculture 116: 353-365.

Beiras R, A Perez \& M Albentosa. 1994. Comparision of the scope for growth with the growth performance of Ostrea edulis seed reared at different food concentrations in an open-flow system. Marine Biology 119: 227-233.

Brett J, J Shelbourn \& C Shoop. 1969. Growth rate and body composition of fingerling sokeye salmon, Oncorhynchus nerka, in relation to temperature and ration size. Journal of Fisheries Research Board of Canada 26: 2363-2394.

Buitrago E, J Buitrago, L Freites \& C Lodeiros. 2009. Identificación de factores que afectan al crecimiento y la supervivencia de la ostra de mangle, Crassostrea rhizophorae (Guilding, 1828), bajo condiciones de cultivo suspendido en la laguna de La Restinga, Isla de Margarita, Venezuela. Zootecnia Tropical 27(1): 79-90.

Buxton C, R Newel \& J Field. 1981. Response-surface analysis of the combined effects of exposure and acclimation temperatures on filtration, oxygen consumption and scope for growth in the oyster Ostrea edulis. Marine Ecology Progress Series 6: 73-82.

Brown J \& B Hartwick. 1988. Influences of temperature, salinity and available food upon suspended culture of the pacific oyster, Crassostrea gigas. I Absolute and allometric growth. Aquaculture 70: 231-251.

Camacho A, U Labarta \& E Navarro. 2000. Energy balance of mussel Mytilus galloprovincialis: the effect of length and age. Marine Ecology Progress Series 199: 149-158.

Chauvaud L, G Thouzeau \& Y Paulet. 1998. Effects of environmental factors on the daily growth rate of Pecten maximus juveniles in the Bay of Brest (France). Journal of Experimental Marine Biology and Ecology 227: 83-111.

Cole F, J Parsons \& C Couturier. 1996. Flow dynamics in and around pearl nets of various mesh sizes. Bulletin of the Aquaculture Association of Canada 3: 77-79.

Cubillo A, L Peteiro, M Fernández \& U Labarta. 2012a. Density-dependent effects on morphological plasticity of Mytilus galloprovincialis in suspended culture. Aquaculture 338-341: 246-252.

Cubillo A, L Peteiro, M Fernández \& U Labarta. 2012b. Influence of stocking density on growth of mussels (Mytilus galloprovincialis) in suspended culture. Aquaculture 342343: 103-111.

Cubillo A, I Fuentes, L Peteiro, M Fernández \& U Labarta. 2012c. Evaluation of self-thinning models and estimation methods in multilayered sessile animal populations. Ecosphere 3(8): 1-23.

Duarte P, M Fernández, R Filgueira \& U Labarta. 2010. Modelling mussel growth in ecosystems with low suspended matter loads. Journal of Sea Research 64: 273286. 
Duarte P, M Fernández \& U Labarta. 2012. Modelling mussel growth in ecosystems with low suspended matter loads using a Dynamic Energy Budget approach. Journal of Sea Research 67: 44-57.

Filgueira R, R Rosland \& J Grant. 2011. A comparison of Scope For Growth (SFG) and Dynamic Energy Budget (DEB) models applied to the blue mussel (Mytilus edulis). Journal of Sea Research 66: 403-410.

Fréchette A \& C Bacher. 1998. A modelling study of optimal stocking density of mussel populations kept in experimental tanks. Journal of Experimental Marine Biology and Ecology 219: 241-255.

Handeland S, A Imsland \& S Stefansson. 2008. The effect of temperature and fish size on growth, feed intake, food conversion efficiency and stomach evacuation rate of Atlantic salmon post-smolts. Aquaculture 283: 36-42.

Hirtle R, M Der Mont \& R O'Dor. 1981. Feeding, growth and metabolic rates in captive short-finned squid, Illex illecebrosus, in relation to the natural population. Journal of Shellfish Research 1: 187-192.

Irisarri J, M Fernández-Reiriz, S Robinson, P Cranford \& U Labarta. 2013. Absortion efficiency of mussels Mytilus edulis and Mytilus galloprovincialis cultured under Integrated Multi-Trophic Aquaculture conditions in the Bay of Fundy (Canada) and Ria Ares-Betanzos (Spain). Aquaculture 388-389: 182-192.

Kooijman S. 2000. Dynamic and energy mass budgets in biological systems, 419 pp. Cambridge University Press, Cambridge.

López J, J Gómez, C Castellanos \& E Acosta. 2010. Efecto de la densidad de siembra sobre el crecimiento y supervivencia de Nodipecten nodosus en un nuevo arte de cultivo. Boletín de Investigaciones Marinas y Costeras 39(1): 177-189.

Marambio J, S Maturana \& B Campos. 2012. Modelo dinámico de crecimiento de la biomasa para Mytilus chilensis en sistemas de cultivo en líneas. Revista de Biología Marina y Oceanografía 47(1): 51-64.

Márquez A, C Lodeiros, D Semidey, M Carpio \& C Graziani. 2011. Crecimiento y supervivencia de la ostra perlífera Pinctada imbricata (Röding 1798), bajo diferentes sistemas de confinamiento en cultivo Suspendido. Zootecnia Tropical 29(3): 337-351.

Monod J. 1942. Recherches sur la croissance des cultures bacteriennes. Actualités Sciéntifiques et Industrielles 911 : 1-212. Hermann et Cie., Paris.

Navarro J \& C Gonzalez. 1998. Physiological responses of the Chilean scallop Argopecten purpuratus to decreasing salinities. Aquaculture 167: 315-327.

Navarro J \& R Thompson. 1996. Physiological energetics of the horse mussel Modiolus modiolus in a cold ocean environment. Marine Ecology Progress Series 138: 135148.
Navarro J \& J Winter. 1982. Ingestion rate, assimilation efficiency and energy balance in Mytilus chilensis in relation to body size and different algal concentrations. Marine Biology 67: 255-266.

Nunes J, J Ferreira, F Gazeau, J Lencart-Silva, X Zhang, M Zhu \& J Fang. 2003. A model for sustainable management of shellfish polyculture in coastal bays. Aquaculture 219: 257-277.

Orellana A. 1998. Modelación de la tasa instantánea de crecimiento del Ostion del Norte (Argopecten purpuratus, Lamarck 1819) en cultivo suspendido. Tesis de Ingeniero en Acuicultura, Facultad de Ciencias del Mar, Universidad Católica del Norte, Coquimbo, 71 pp.

Paloheimo J \& L Dickie. 1965. Food and growth of fishes. I. A growth curve derived from experimental data. Journal of Fisheries Research Board of Canada 22: 521-542.

Paloheimo J \& L Dickie. 1966. Food and growth of fishes. II. Effects of food and temperature on the relation between metabolism and body weigth. Journal of Fisheries Research Board of Canada 23: 869-908.

Parsons T, M Takahashi \& B Hargrave. 1995. Biological oceanographic processes, 330 pp. Pergamon, New York.

Penney R \& T Mills. 1996. Effect of spat grading and net mesh size on the growth and survival of juvenile cultured sea scallop, Placopecten magellanicus, in Newfoundland. Bulletin of the Aquaculture Association of Canada 3: 8082.

Pilditch C, J Grant, A Mellet, C Carver \& P Cranford. 1996. Seston supply to scallop in suspended culture. Journal of Shellfish Research 15: 504.

Pütter A. 1920. Studien über physiologische Ähnlichkeit VI. Wachstumsähnlichkeiten. Pflügers Archiv European Journal of Physiology 180: 298-340.

Raillard O \& A Ménesguen. 1994. An ecosystem box model for estimating the carrying of a macrotidal shellfish system. Marine Ecology Progress Series 117: 117-130.

Rheault R \& A Rice. 1993. Food limited growth and condition index in Crassostrea virginica and Argopecten irradians. Journal of Shellfish Research 12: 113.

Ricker W. 1958. Handbook of computation for biological statistics of fish populations. Bulletin of Fisheries Research Board of Canada 119: 1-300.

Riisgard H \& A Randlov. 1981. Energy budgets, growth and filtration rates in Mytilus edulis at different algal concentrations. Marine Biology 61: 227-234.

Rosemberg R \& L Loo. 1983. Energy flow in a Mytilus edulis culture in Western Sweden. Aquaculture 35: 151-161.

Rosland R, Ø Strand, M Alunno-Bruscia, C Bacher \& T Strohmeier. 2009. Applying Dynamic Energy Budget (DEB) theory to simulate growth and bio-energetics of blue mussels under low seston conditions. Journal of Sea Research 62: 49-61. 
Rueda J, A Smaal \& H Scholten. 2005. A growth model of the cockle (Cerastoderma edule L.) tested in the Oosterschelde estuary (The Netherlands). Journal of Sea Research 54: 276-298.

Scholten H \& A Smaal. 1998. Responses of Mytilus edulis L. to varying food concentrations: testing EMMY, an ecophysiological model. Journal of Experimental Marine Biology and Ecology 219: 217-239.

Selong J, T McMahon, A Zale \& F Barrows. 2001. Effect of temperature on growth and survival of Bull Ttrout, with application of an improved method for determining thermal tolerance in fishes. Transactions of the American Fisheries Society 130: 1026-1037.

Springborn R, A Jensen \& W Chang. 1994. A variable growth rate modification of Von Bertalanffy equation for aquaculture. Aquaculture Research 25: 259-267.

Talbot C. 1994. How growth relates to ration size. Fish Farmer 17: 1-3.

Taylor J, R Rose \& P Southgate. 1997. Fouling animals and their effect on the growth of the silver-lip pearl oysters Pinctada maxima (Jameson), held in suspended nursery culture. Aquaculture 153: 31-40.

Taylor J, R Rose, C Taylor \& P Southgate. 1997. Effects of stocking density on growth and survival of early juvenile silver-lip pearl oysters, Pinctada maxima (Jameson), held in suspended nursery culture. Aquaculture 153: 41-49.

Troost T, J Wijsman, S Saraiva \& V Freitas. 2010. Modelling shellfish growth with dynamic energy budget models: an application for cockles and mussels in the Oosterschelde (southwest Netherlands). Philosophical Transactions of the Royal Society B 365: 3567-3577.
Urban E, J Gary, D Pruder \& C Langdon. 1983. Effects of ration on growth and growth efficency of juveniles of Crassostrea virginica. Journal of Shellfish Research 3: 5157.

Ursin E. 1967. A mathematical model of some aspects of fish growth, respiration, and mortality. Journal of Fisheries Research Board of Canada 24: 2355-2453.

Van der Meer J. 2006. An introduction to dynamic energy budget (DEB) models with emphasis on parameter estimation. Journal of Sea Research 56: 85-102.

Van der Veer H \& M Alluno-Bruscia. 2006. The DEBIB project: Dynamic Energy Budgets in Bivalves. Journal of Sea Research 56: 81-84.

Von Bertalanffy L. 1938. A quantitative theory of organics growth (Inquires on growth laws II). Human Biology 10: 181-213.

Widdows J. 1978. Physiological indices of stress in Mytilus edulis. Journal of the Marine Biological Association of the United Kingdom 58: 125-142.

Widdows J, P Fieth \& C Worral. 1979. Relationships between seston, available food and feeding activity in the common mussel Mytilus edulis. Marine Biology 50: 195-207.

Winter J. 1977. Suspension-feeding in lamellibranchiate bivalves, with particular reference to Aquaculture. Medio Ambiente 3: 48-69.

Xie S, Y Cui, Y Yang \& J Lin. 1997. Energy budget of Nile tilapia (Oreochromis niloticus) in relation to ration size. Aquaculture 154: 57-68.

Yi Y. 1998. A bioenergetic growth model for Nile tilapia (Oreochromis niloticus) based on limiting nutrient and fish standing crop in fertilized ponds. Aquacultural Engineering 18: $157-173$

Recibido el 11 de marzo de 2013 y aceptado el 16 de enero de 2014

Editor: Claudia Bustos D. 